\title{
PREVALÊNCIA DO ALEITAMENTO MATERNO NO BRASIL SEGUNDO CONDIÇÕES SOCIOECONÔMICAS E DEMOGRÁFICAS
}

\author{
PREVALENCE OF BREASTFEEDING IN BRAZIL \\ ACCORDING TO SOCIOECONOMIC AND DEMOGRAPHICS \\ CONDITIONS
}

Daniela Wenzel $^{1}$
Sônia Buongermino de Souza ${ }^{2}$

Wenzel D e Souza SB. Prevalência do aleitamento materno no Brasil segundo condições socioeconômicas e demográficas. Rev Bras Cresc e Desenv Hum 2011; 21(2): 251-258.

\section{RESUMO}

Objetivo: quantificar a frequência do Aleitamento Materno (AM) no Brasil, macro-regiões e áreas urbanas e rurais, segundo variáveis socioeconômicas e demográficas. Metodo: amostra de 2958 crianças, de zero a um ano de idade, representativas da população brasileira. Os dados são da Pesquisa de Orçamentos Familiares-POF, 2002-2003. Utilizaram-se para análise, com tabelas de contingência, os testes qui-quadrado e de tendência para verificar as relações entre as variáveis com significância de 5\%. Resultados: no grupo de crianças com até 180 dias de idade, a frequência da amamentação foi de $58 \%$. Nas regiões, foram $63 \%, 59 \%, 51 \%, 61 \%$ e $56 \%$, respectivamente para Norte, Nordeste, Sudeste, Sul e Centrooeste. Na área rural foi de $60 \%$ e na urbana $58 \%$. Mães com maior renda e maior escolaridade apresentaram $60 \%$ e $65 \%$, respectivamente. No grupo com mais de 180 dias a frequência no Brasil foi de 35\%. Nas regiões foram: $44 \%, 34 \%, 37 \%, 34 \%, 28 \%$, respectivamente para Norte, Nordeste, Sudeste, Sul e Centro-oeste. Na área rural foi de 39\% e na urbana de $34 \%$. Mães com menor renda e menor escolaridade apresentaram 38\% e 40\%, respectivamente. Conclusão: as maiores prevalências foram verificadas nas regiões Norte e Nordeste do País. Os padrões de AM diferiram quanto à faixa etária. No grupo de crianças com até 180 dias, mães com maior renda e escolaridade apresentaram maior frequência da amamentação. Por outro lado, no grupo com mais de 180 dias, a prevalência foi maior entre mães de menor renda e escolaridade.

Palavras-chave: aleitamento materno; prevalência; indicadores socioeconômicos.

Departamento de Merenda Escolar da Secretaria de Educação do Município de São Paulo, Brasil.

2 Departamento de Nutrição da Faculdade de Saúde Pública da Universidade de São Paulo (USP), Brasil.

Correspondência para: daniwenzel@usp.br

Este trabalho foi baseado na tese de doutorado intitulada "Aleitamento Materno: estudo nacional da prevalência e determinantes no Brasil, nas macro-regiões e áreas urbanas e rurais”, realizado junto ao Departamento de Nutrição da Faculdade de Saúde Pública da Universidade de São Paulo em 2008. 


\begin{abstract}
Objective: this study aimed to quantify the prevalence of breastfeeding in Brazil, macroregions, urban and rural areas, according to socioeconomic and demographic conditions. Methods: the sample consisted of 2,958 Brazilian children from 0 to 1 year old. Data are from the Search Family Budgets, 2002-2003. Contingency tables, the chisquare test and trend test were used in order to verify the relationship between variables, significance was set at 5\% and prevalence curves of breastfeeeding were made. Results: the medians of brestfeeding (BF) duration were 8 months for the North region, 9 months for Northeast, Southeast, and South and 10 months for Midwest. In the group 0 to 6 months, the frequency of $\mathrm{BF}$ in Brazil was $58 \%$. We found $63 \%, 59 \%, 51 \%, 61 \%$ and $56 \%$, respectively, for North, Northeast, Southeast, South and the Midwest. In the rural area we found $60 \%$ and $58 \%$ in urban area. Mothers with higher incomes and more education had $60 \%$ and $65 \%$, respectively. In the group 7 to 12 months the prevalence in Brazil was $35 \%$. We found: $44 \%, 34 \%, 37 \%, 34 \%, 28 \%$, respectively, for North, Northeast, Southeast, South and the Midwest. In the rural area we reported 39\% and 34\% for urban area. Mothers with lower incomes and less education had $38 \%$ and $40 \%$ of BF frequency, respectively. Conclusion: the greatest prevalence was found in North and Northeast region and the greatest median of BF was found in Midwest region. The BF patterns differed in two age groups. In the group 0-6 months, mothers with higher income and more education had higher frequency of BF. However, in the group of children from 7 to 12 months, the prevalence of BF was higher among mothers with lower income and less education.
\end{abstract}

Key words: breastfeeding; prevalence; socioeconomic indicators.

\section{INTRODUÇÃO}

A superioridade da amamentação como fonte de nutrientes, de proteção contra doenças e de afeto faz com que especialistas do mundo inteiro o recomendem. Alguns estudos têm mostrado seus benefícios para a saúde da criança e da mulher, para o fortalecimento do vínculo afetivo entre mãe e filho, bem como na economia para famílias, instituições de saúde, governos e nações, entre outros aspectos ${ }^{1,2}$.

Estudos realizados no Brasil e no mundo mostram que a amamentação ajuda a prevenir tanto a desnutrição como a obesida$\mathrm{de}^{3,4,5,6,}$, além de doenças como a diarreia e infecções respiratórias, que são causas importantes de mortalidade infantil ${ }^{7,8,9}$.

Devido sua importância e seus benefícios, a Organização Mundial da Saúde preconiza aleitamento materno exclusivo (AME) até seis meses de vida, com a complementação de outros alimentos a partir do sexto mês até pelo menos dois anos de idade ${ }^{10}$.

No Brasil, apesar da escassez de dados representativos que reflitam a situação da amamentação, estudos regionais mostram que, até a década de 70 (nas décadas de 60 e 70), a prática do aleitamento sofreu considerável declínio ${ }^{11}$.

Posteriormente, alguns trabalhos sobre aleitamento materno, com representatividade nacional da população, foram realizados no Brasil possibilitando acompanhar a situação da amamentação no país. No primeiro deles, o Estudo Nacional de Despesa Familiar ENDEF $^{12}$, realizado em 1974-1975, as porcentagens de crianças amamentadas aos seis e doze meses foram de $33 \%$ e $23 \%$, respectivamente. Em 1989, nessas mesmas idades, foram de 49\% e 37\%, respectivamente, segundo dados da Pesquisa Nacional sobre Saúde e Nutrição $\mathrm{PNSN}^{13}$. Em 1996, segundo a Pesquisa Nacio- 
nal sobre Demografia e Saúde - PNDS $^{14}$, a prevalência da amamentação, até seis meses, foi de $51 \%$ e na faixa etária de 10 a 14 meses foi de $37 \%$. Em 2006, em uma nova versão da PNDS $^{15}$, os valores foram de $91,8 \%$ para crianças de zero a seis meses e de $60,8 \%$ entre crianças de sete a dez meses de idade. Em 1999, segundo a pesquisa "Prevalência de aleitamento materno nas capitais brasileiras e no Distrito Federal" realizada pelo Ministério da Saúde, nas áreas urbanas das capitais brasileiras ${ }^{16}$, a prevalência no grupo de zero a seis meses foi de $66,8 \%$ e no grupo de nove a doze meses, foi de $42,4 \%$. Já em 2008, na nova versão dessa pesquisa, a prevalência de aleitamento materno foi de $67,7 \%$ em todo país, entre as crianças menores de um ano. Comparando os grupos de nove a doze meses, nos dois momentos, a prevalência de AM aumentou 42,4 para $58,7 \%$.

O conhecimento sobre as tendências nacionais e regionais da amamentação é de fundamental importância, pois permite conhecer suas diferenças e embasar mudanças e ajustes nas práticas de promoção e incentivo ao aleitamento materno ${ }^{17}$.

Assim, o objetivo é descrever a prevalência do aleitamento materno no Brasil nas macro-regiões e nas áreas urbanas e rurais segundo indicadores socioeconômicos em crianças até um ano de idade.

\section{MÉTODO}

A Pesquisa de Orçamentos Familiares (POF), realizada em 2002-2003 pelo Instituto Brasileiro de Geografia e Estatística (IBGE) é um estudo com representação nacional, de base domiciliar, com uma complexa seleção amostral em múltiplos estágios envolvendo estratificação e conglomerados. A POF foi composta por uma amostra de 48.470 famílias. A estratificação geográfica permitiu a representatividade de todo o território nacional (regiões Norte, Nordeste, Sudeste, Sul e Centro Oeste), além de áreas urbanas e rurais. A amostragem foi descrita em publicação do IBGE de $2004^{18}$.

A POF não tinha, em seu instrumento de coleta de dados, questões sobre aleitamento materno. No entanto, ao classificar o sexo dos moradores nos domicílios da amostra, se atribuiu às mulheres três categorias: feminino, gestante e lactante. Foram verificados todos os domicílios com crianças menores de um ano. A amostra foi composta por 2958 crianças, sendo 1477 de zero a 180 dias e 1481 crianças com 181 a 365 dias de idade.

A variável dependente foi aleitamento materno (sim, não) e as independentes: idade materna $(<20,20-25,26-30,>30$ anos; escolaridade materna $(<4$ anos, 5-8 anos, 9 anos ou mais); renda domiciliar per capita (quartis de renda); região (Norte, Nordeste, Sudeste, Sul e Centro-Oeste); e tipo de área (rural e urbana).

Usando a categoria de lactante foi possível verificar o número de crianças menores de um ano de idade em aleitamento materno na população. Pela inexistência de dados sobre a alimentação infantil não foi possível especificar o tipo de aleitamento, ou seja, se estavam ou não recebendo, além do leite materno, outro tipo de alimento líquido, sólido, ou mesmo, qualquer outro tipo de leite.

\section{Análise estatística}

As macro-regiões foram caracterizadas segundo fatores sociais, econômicos e demográficos. As prevalências médias de aleitamento materno foram estimadas por ponto e por intervalo com $95 \%$ de confiança, de acordo com as categorias das variáveis independentes, apresentadas em tabelas para os grupos etários zero a 180 dias e 181 a 365 dias. Utilizou-se teste qui-quadrado (global e de tendência) para as comparações das prevalências, com nível de significância de 5\%. As análises foram realizadas com o pacote estatístico SPSS 13 e o Stata.09. 
Este estudo está de acordo com as normas da Resolução ${ }^{\circ} 196$ de 10/10/1996 do Conselho Nacional de Saúde e foi aprovado pelo Comitê de Ética em Pesquisa da Faculdade de Saúde Pública da Universidade de São Paulo.

\section{RESULTADOS}

Foi constatado que $74 \%$ dos indivíduos da amostra viviam em áreas urbanas do país, a média de escolaridade materna foi de seis anos e três meses, a idade média das mães foi de 26 anos, a média da renda em todo o Brasil foi de $\mathrm{R} \$ 1000,00$.

Nas regiões Norte, Nordeste e Centrooeste do país, cerca de 50\% das mães eram jo- vens, com até 25 anos de idade. Os níveis mais baixos de escolaridade foram verificados entre as mães do Norte e do Nordeste com, respectivamente, $70,6 \%$ e $73,0 \%$ com até oito anos de estudo. O mesmo pode ser observado em relação à renda, pois $55,1 \%$ e $65,6 \%$ das mães, respectivamente do Norte e do Nordeste, tinham até o segundo quarto de renda.

$\mathrm{Na}$ região Centro-oeste, a maior proporção das mães também era mais jovem, porém apresentou maior nível de escolaridade e maior nível de renda, em relação às do Norte e Nordeste. As regiões Sul e Sudeste apresentaram maior proporção de mães mais velhas, com maior nível de escolaridade e maior nível de renda (Tabela 1).

Considerando-se os dois grupos etários, verifica-se que a região do Brasil que apresen-

Tabela 1: Características da população brasileira nas regiões do Brasil, 2002 -2003

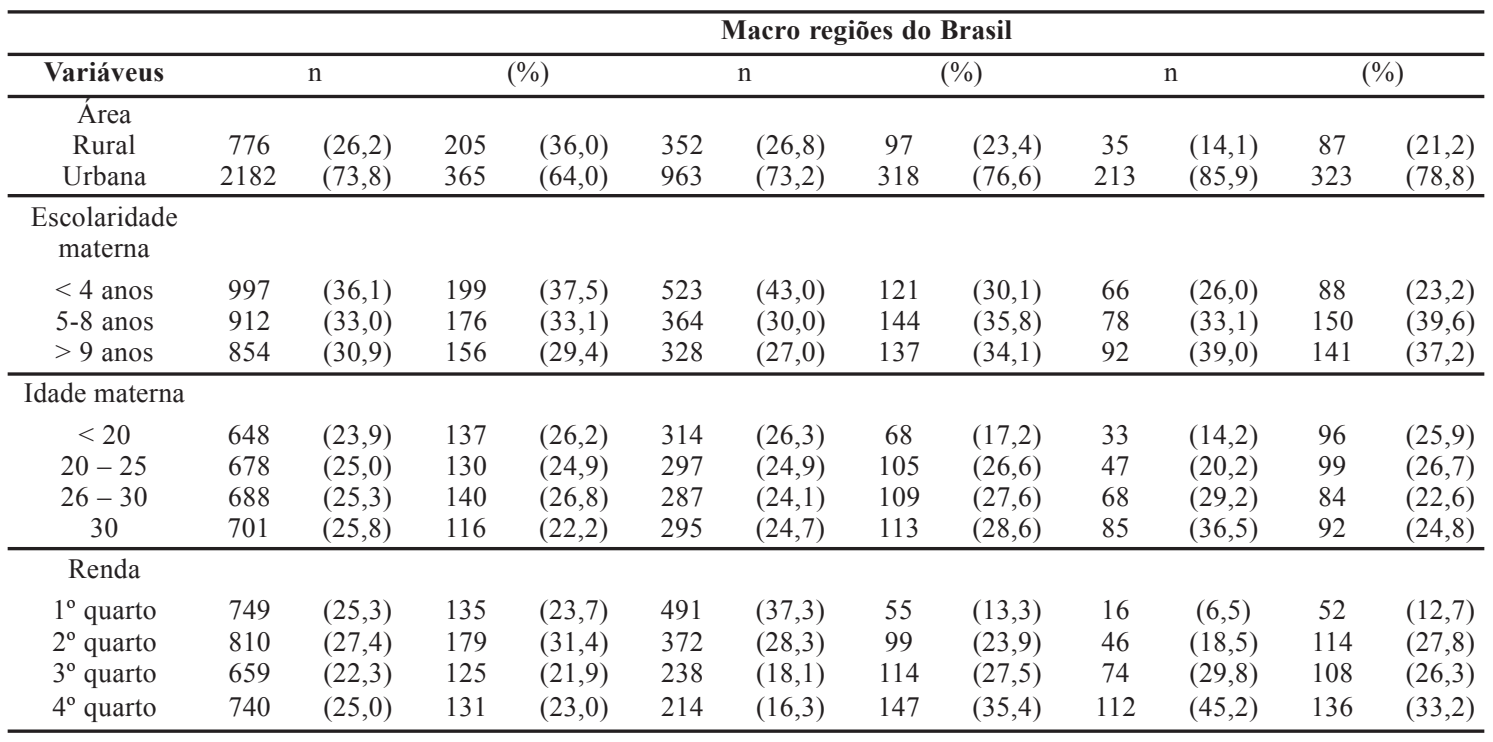

tou maior prevalência do aleitamento, no grupo de crianças de zero a 180 dias de idade, foi a região Norte e a menor foi a região Sudeste do país (Tabela 2). A área rural apresentou maior prevalência comparada à urbana. As diferenças verificadas entre as prevalências nas regiões, bem como nas áreas urbanas e rurais, nessa faixa etária, não foram probabilistica- mente significantes, $\left(\mathrm{c}^{2}=8,082\right.$ e $\left.\mathrm{p}=0,090\right)$, $\left(\mathrm{c}^{2}=0,417\right.$ e $\left.\mathrm{p}=0,518\right)$, respectivamente.

A região do Brasil que apresentou maior prevalência de aleitamento, na faixa etária de 181 a 365 dias, foi a região Norte e a menor foi observada na região Centro-oeste (Tabela 2). Ao contrário do verificado com o grupo de zero a 180 dias, neste grupo as diferenças entre as 
Tabela 2: Prevalência do aleitamento materno em crianças de zero a um ano, nas macro-regiões, áreas rurais e urbanas e Brasil, 2002-2003

\begin{tabular}{|c|c|c|c|c|c|c|c|c|}
\hline & \multicolumn{8}{|c|}{ Aleitamento Materno } \\
\hline & \multicolumn{3}{|c|}{ Zero a 180 dias } & \multicolumn{5}{|c|}{181 a 365 dias } \\
\hline & $\mathrm{n}$ & $\%$ & IC $95 \%$ & $(p)^{*}$ & $\mathrm{n}$ & $\%$ & IC $95 \%$ & $(p)^{*}$ \\
\hline Regiões do Brasil & & & & 0,090 & & & & 0,004 \\
\hline Norte & 290 & $63 \%$ & $57 \%-68 \%$ & & 280 & $44 \%$ & $38 \%-49 \%$ & \\
\hline Nordeste & 660 & $59 \%$ & $55 \%-62 \%$ & & 655 & $34 \%$ & $30 \%-37 \%$ & \\
\hline Sudeste & 200 & $51 \%$ & $43 \%-57 \%$ & & 215 & $37 \%$ & $31 \%-44 \%$ & \\
\hline Sul & 118 & $61 \%$ & $52 \%-70 \%$ & & 130 & $34 \%$ & $26 \%-42 \%$ & \\
\hline Centro-Oeste & 209 & $56 \%$ & $48 \%-62 \%$ & & 201 & $28 \%$ & $21 \%-34 \%$ & \\
\hline Áreas do Brasil & & & & 0,518 & & & & 0,106 \\
\hline Urbana & 1104 & $58 \%$ & $55 \%-61 \%$ & & 1078 & $34 \%$ & $31 \%-37 \%$ & \\
\hline Rural & 373 & $60 \%$ & $55 \%-65 \%$ & & 403 & $39 \%$ & $34 \%-43 \%$ & \\
\hline Brasil & 1477 & $58 \%$ & $55 \%-60 \%$ & & 1481 & $35 \%$ & $33 \%-38 \%$ & \\
\hline
\end{tabular}

(*) Valores de p: teste qui-quadrado. (*) P-values: chi-square

prevalências de amamentação, nas macro-regiões, foram probabilisticamente significantes $\left(\mathrm{x}^{2}=15,250 \mathrm{e} \mathrm{p}=0,004\right)$. Entre as áreas urbanas e rurais, as diferenças não foram significantes $\left(\mathrm{x}^{2}=2,616\right.$ e $\left.\mathrm{p}=0,106\right)$.

Em relação às variáveis independentes (tabela 3), no grupo de zero a 180 dias de idade as maiores prevalências de aleitamento materno foram encontradas entre crianças de mães com nove anos ou mais de escolaridade, com maior renda ( $3^{\circ}$ e $4^{\circ}$ quartos), e com menos de trinta anos de idade, e entre as que viviam em áreas rurais do país. No grupo de 181 a 365 dias de idade, o quadro foi justamente o oposto, com maiores prevalências de aleitamento materno entre crianças de mães com menos de quatro anos de escolaridade, com menor renda ( $1^{\circ}$ e $2^{\circ}$ quartos), mas, também, entre as com idades abaixo dos trinta anos e vivendo em áreas rurais (tabela 3).

Tabela 3: Prevalência de AM em crianças de zero a 180 dias e de 181 a 365 dias de idade, segundo determinantes socioeconômicos e demográficos, Brasil, 2002- 2003

\begin{tabular}{|c|c|c|c|c|c|c|c|c|}
\hline & \multicolumn{8}{|c|}{ Aleitamento Materno } \\
\hline & \multicolumn{3}{|c|}{ Zero a 180 dias } & \multicolumn{5}{|c|}{181 a 365 dias } \\
\hline & Não & sim & $\%$ & IC $95 \%$ & não & $\operatorname{sim}$ & $\%$ & IC $95 \%$ \\
\hline \multicolumn{9}{|l|}{ Escol. Materna } \\
\hline$<=4$ anos & 197 & 284 & $59 \%$ & $(55-63)$ & 310 & 206 & $40 \%$ & $(35-44)$ \\
\hline 5 a 8 anos & 185 & 286 & $61 \%$ & $(56-65)$ & 275 & 166 & $38 \%$ & $(33-42)$ \\
\hline 9 anos e mais & 157 & 288 & $65 \%$ & $(60-69)$ & 260 & 149 & $36 \%$ & $(32-41)$ \\
\hline \multicolumn{9}{|l|}{ Renda } \\
\hline $1^{\circ}$ quarto & 162 & 216 & $57 \%$ & $(52-62)$ & 229 & 142 & $38 \%$ & $(33-43)$ \\
\hline $2^{\circ}$ quarto & 177 & 229 & $56 \%$ & $(52-61)$ & 263 & 141 & $35 \%$ & $(30-39)$ \\
\hline $3^{\circ}$ quarto & 132 & 194 & $60 \%$ & $(54-65)$ & 220 & 113 & $34 \%$ & $(29-39)$ \\
\hline $4^{\circ}$ quarto & 148 & 219 & $60 \%$ & $(55-65)$ & 248 & 125 & $33 \%$ & $(29-38)$ \\
\hline \multicolumn{9}{|l|}{ Idade Materna* } \\
\hline$<=20$ anos & 127 & 232 & $65 \%$ & $(60-70)$ & 175 & 114 & $39 \%$ & $(34-45)$ \\
\hline 21 a 25 anos & 126 & 204 & $62 \%$ & $(57-67)$ & 207 & 141 & $40 \%$ & $(35-46)$ \\
\hline 26 a 30 anos & 115 & 209 & $65 \%$ & $(59-70)$ & 233 & 131 & $36 \%$ & $(31-41)$ \\
\hline$>30$ anos & 163 & 202 & $54 \%$ & $(50-60)$ & 212 & 124 & $37 \%$ & $(32-42)$ \\
\hline \multicolumn{9}{|l|}{ Área } \\
\hline Urbana & 468 & 636 & $57 \%$ & $(54-60)$ & 712 & 366 & $34 \%$ & $(31-37)$ \\
\hline Rural & 151 & 222 & $59 \%$ & $(54-64)$ & 248 & 155 & $39 \%$ & $(33-43)$ \\
\hline
\end{tabular}

* Teste de tendência 


\section{DISCUSSÃO}

A necessidade de usar a categoria de Lactante para obter os dados de aleitamento materno pode ter sido uma limitação do presente estudo, uma vez que tratam-se de dados obtidos indiretamente. Entretanto, como o presente estudo foi realizado com amostra da Pesquisa de Orçamentos Familiares, garantiu-se a confiabilidade dos resultados, pois o planejamento e execução da POF foram elaborados de modo a minimizar vieses de amostragem e de aferição das informações.

A amostra obtida para este trabalho é de tamanho adequado e representativo da população nacional. Os resultados encontrados possivelmente refletem a situação da prática do aleitamento materno nas idades estudadas, na época investigada.

Entre as regiões do país, a Norte foi a que apresentou maior prevalência de aleitamento materno nas duas faixas etárias. A menor prevalência, no grupo de zero a 180 dias, foi encontrada na região Sudeste. No grupo de 181 a 365 dias, a prevalência mais baixa foi observada na região Centro-oeste.

Verifica-se que, nos dois grupos de idade, a prevalência de aleitamento materno ainda é maior nas áreas rurais do país. Alguns outros trabalhos também mostram que entre mães residentes em áreas rurais, por apresentarem situação mais favorável para amamentar, as prevalências de aleitamento materno são maiores, comparadas às de áreas urbanas ${ }^{19,20}$.

No Brasil, as prevalências continuam maiores nas áreas rurais, do que nas urbanas, porém a diferença entre elas vem diminuindo substancialmente. Em crianças de zero a 180 dias a diferença entre as áreas urbanas e rurais foi de 25 pontos percentuais em 1975 segundo ENDEF $^{12}, 10$ pontos percentuais em 1989 segundo $\mathrm{PNSN}^{13}$ e dois pontos percentuais segundo os dados deste estudo.

No grupo de crianças de 181 a 365 dias, a amamentação também foi maior nas áreas rurais do país. Mas nessa faixa etária foi possível detectar outra realidade. Na área urbana, em $1975^{12}$, a prevalência do aleitamento materno foi de $13 \%$; em $1989^{13}$ foi de $34 \%$, valor que permaneceu até 2002 - 2003. Na área rural, em 1975 a prevalência foi de 34\%, em 1989 de $41 \%$ e em 2002-2003, com dados deste estudo, observou-se prevalência de $39 \%$. A diferença entre as duas áreas, que foi de 21 pontos percentuais em 1975, baixou para sete pontos percentuais em 1989 e, em 2002-2003, encontrava-se em cinco pontos percentuais.

Ainda que o aumento da amamentação fosse menos expressivo nas áreas rurais, o aleitamento materno nessas áreas nunca chegou a alcançar níveis tão baixos como na área urbana. Segundo Venâncio e Monteiro ${ }^{11}$, isto pode indicar que houve, de certa forma, uma reversão da tendência esperada de assimilação dos valores culturais dominantes, por parte dos estratos menos favorecidos das populações, ou seja, a população rural ainda não incorporou os valores da população urbana.

Essa diminuição na diferença entre as prevalências de aleitamento materno nas áreas urbanas e rurais pode, também, ser explicada em função do êxodo populacional da área rural para a urbana, como já previa Perez Escamilla $^{19}$. Essa situação faz com que se pense na necessidade de rever a efetividade dos programas de promoção do Aleitamento Materno.

Dois fatores precisam ser destacados: primeiro a diminuição entre a diferença das prevalências nas áreas urbanas e rurais. Observou-se de maneira geral que, embora ainda haja prevalência maior de aleitamento materno nas áreas rurais do país, nos dois grupos de idade, essa situação tende a se modificar em breve. Segundo: não houve acréscimo na prevalência do aleitamento materno, em crianças de 181 a 365 dias, nas duas áreas.

Pode-se sugerir a hipótese de que, na área rural, a permanência de padrões culturais tradicionais ou a persistência de estruturas de 
apoio familiar facilitem a manutenção do aleitamento materno. Outro aspecto relevante, ligado ao âmbito geográfico, é que a existência de padrões de amamentação diferentes por áreas, pode ocorrer, também, devido à presença de diferentes estruturas nos serviços e programas de saúde.

Isto demonstra que, embora a prática de aleitamento materno seja frequente em todo o país, o seu abandono ainda é precoce, em todas as regiões, principalmente quando são estudadas crianças já em fase de complementação alimentar, ou seja, com mais de seis meses. Atenção é necessária, uma vez que se espera que as crianças sejam amamentadas até pelo menos dois anos de idade segundo a recomendação da Organização Mundial da Saúde, $2004^{10}$.

Em relação aos indicadores socioeconômicos, neste estudo, verificaram-se cenários opostos entre as faixas de idade das crianças. As mães com renda mais alta e maior escolaridade apresentaram maior frequência da amamentação entre crianças de zero a 180 dias de idade. Por outro lado, no grupo com mais de 180 dias, a prevalência foi maior entre mães de menor renda e com menos de quatro anos de escolaridade.

Supõe-se que essa associação seja devido ao fato de a condição econômica favorável da família e a alta escolaridade materna facili-

\section{REFERÊNCIAS}

1. Araújo MFM, Del Fiaco A. Pimentel LS. et al. Custo e economia da prática do aleitamento materno para a família. Rev. Bras. Saúde Materno Infant. 2004; 4(2): 135-141.

2. Ergenekon-Ozelci P, Elmaci N, Ertem M, Saka G. Breastfeeding beliefs and practices among migrant mothers in slums of Diyarbakir, Turkey 2001. Eur J Public Health. 2006; 16(2):143-8.

3. Kries RV, Koletzko B, Sauerwald T, Mutius EV, Barnert D, Veit Grunert, et al. Breast feeding and obesity: cross sectional study. BMJ 1999; 319: 147-150. tarem o acesso à informação, o que pode ter favorecido o oferecimento de leite humano aos seus filhos. Também, parece existir maior valorização dos benefícios do aleitamento materno nas classes mais favorecidas. Ainda reforçando esta hipótese, acredita-se que mães com maior renda e maior escolaridade, ao final do período de licença maternidade, voltem a ocupar sua função no mercado de trabalho, deixando de amamentar, ocasionando a maior prevalência do aleitamento no grupo com menor renda, no segundo semestre de vida.

Em estudo realizado com 1057 crianças menores de seis meses no município do Rio de Janeiro, os resultados apontaram para uma introdução precoce de leite não materno entre as mães com menos de oito anos de estudo ${ }^{21}$. Em outro estudo realizado com 12.917 crianças, a prevalência de amamentação foi maior em crianças com até seis meses, filhos de mães com nível econômico elevado ( $4^{\circ}$ quartil de renda $)^{22}$.

Aumentar a prevalência do aleitamento materno é objetivo primordial da saúde pública, especialmente entre grupos que são menos prováveis de iniciar e sustentar a amamentação. Dessa forma, os resultados deste estudo remetem para a preocupação, já existente, de se pensar em estratégias de atuação que causem impactos, também, no aleitamento de crianças com idades acima de seis meses.

4. Toschke AM, Thorsteinsdottir KH, Kries RV Meal frequency, breakfast consumption and childhood obesity. Int $J$ Pediatr Obes. 2009; 21:1-7.

5. Schack-Nielsen L, Sørensen TIa, Mortensen EL, Michaelsen KF. Late introduction of complementary feeding, rather than duration of breastfeeding, may protect against adult overweight. Am J Clin Nutr. 2010; 91(3):619-27.

6. Kramer MS. Breastfeeding, complementary (solid) foods, and long-term risk of obesity. Am J Clin Nutr. 2010; 91(3): 500-1.

7. Escuder MML, Venâncio SY, Pereira JCR. Estimativa de impacto da amamentação 
sobre a mortalidade infantil. Rev Saúde Pública. 2003; 37(3):319-25.

8. Victora CG, Behague DP, Barros FC, Olinto MT, Weiderpass E. Pacifier use and hort breastfeeding duration: cause, consequence or coincidence? Pediatrics. 1997; 99:445-53

9. Mihrshahi S, Ichikawa N, Shuaib M, Oddy W, Ampon R, Dibley MJ, et al. Prevalence of exclusive breastfeeding in Bangladesh and its association with diarrhoea and acute respiratory infection: results of the multiple indicator cluster survey 2003. $J$ Health Popul Nutr. 2007; 25(2):195-204.

10. World Health Organization. Evidence for the ten steps to successful breastfeeding Rev.ed. Division of Child Health and Development. 2004.

11. Venâncio SI, Monteiro CA. A tendência da prática da amamentação no Brasil nas décadas de 70 e 80 . Rev. Bras. Epidemiol. 1998; 1:40-49.

12. IBGE. Metodologia do Estudo Nacional da Despesa Familiar - ENDEF 75. Rio de Janeiro: Fundação IBGE; 1983.

13. INSTITUTO NACIONAL DE ALIMENTAÇÃO A NUTRIÇÃO. Pesquisa Nacional sobre saúde e Nutrição. Brasília, DF; 1990

14. Pesquisa Nacional sobre Demografia e Saúde. Rio de Janeiro: BEMFAM/DHS/ IBGE/MS/ UNICEF, PNDS, 1997.

15. Pesquisa Nacional sobre Demografia e Saúde. Relatório final. Rio de Janeiro:
BEMFAM/DHS/IBGE/MS/ UNICEF, PNDS, 2008.

16. Brasil, Ministério da Saúde, Secretaria de Políticas de Saúde, Área de Saúde da Criança. Prevalência de aleitamento materno nas capitais brasileiras e no Distrito Federal. Brasília: Ministério da Saúde; 2001.

17. Kummer SC, Giugliani ERJ, Susinc LO, Follettod JL, et al. Evolução do padrão de aleitamento materno. Rev Saúde Pública. 2000; 34(2):143-8.

18. Instituto Brasileiro de Geografia e Estatística (IBGE). Pesquisa de orçamentos familiares, 2002-2003. Aquisição alimentar domiciliar per capita, Brasil e grandes regiões. Rio de Janeiro; 2004.

19. Pérez-Escamilla R. Breastfeeding and the nutritional transition in the Latin American and Caribbean Region: a success story? Cad. Saúde Pública. 2003; 19(Sup.1): S119-S127.

20. Suárez Gil P, Alonso Lorenzo JC, López Díaz JL, Martín Rodríguez D, Martínez Suárez MM. Prevalencia y duración de la lactancia materna en Asturias. Gac Sanit. 2000; 15 (2): 104-110.

21. Niquini R P, Bittencourt S A, Lacerda E M A, Leal M C. Fatores associados à introdução precoce de leite artificial, Município do Rio de Janeiro, 2007. Rev. bras. epidemiol. 2009; 12 (3): 446-457.

22. Wallby T, Hjern A. Region of birth, income and breastfeeding in a Swedish county. Acta Paediatr. 2009; 98(11):1799-804.

Recebido em: 20/nov./2010

Modificado em 18/jan./2011 Aceito em 26/mar./2011 